\title{
Reappraisal of role of angiotensin receptor blockers in cardiovascular protection
}

This article was published in the following Dove Press journal:

Vascular Health and Risk Management

I3 May 201 I

Number of times this article has been viewed

\section{Venkata S Ram}

Texas Blood Pressure Institute, Clinical Research Institute of Dallas Nephrology Associates; and Department of Internal Medicine, University of Texas Southwestern Medical Center at Dallas, Dallas, TX, USA
Correspondence: C Venkata S Ram University of Texas Southwestern Medical Center at Dallas, 1420 Viceroy Drive, Dallas, TX 75235, USA

Tel + I 2/43582300

Fax + I 2143666027

Email ramv@dneph.com
Abstract: Angiotensin-converting enzyme inhibitors (ACEIs) and angiotensin receptor blockers (ARBs) have shown cardioprotective and renoprotective properties. These agents are recommended as first-line therapy for the treatment of hypertension and the reduction of cardiovascular risk. Early studies pointed to the cardioprotective and renoprotective effects of ARBs in high-risk patients. The ONgoing Telmisartan Alone and in combination with Ramipril Global Endpoint Trial (ONTARGET) established the clinical equivalence of the cardioprotective and renoprotective effects of telmisartan and ramipril, but did not find an added benefit of the combination over ramipril alone. Similar findings were observed in the Telmisartan Randomized AssessmeNt Study in aCE INtolerant subjects with cardiovascular Disease (TRANSCEND) trial conducted in ACEI-intolerant patients. In ONTARGET, telmisartan had a better tolerability profile with similar renoprotective properties compared with ramipril, suggesting a potential clinical benefit over ramipril. The recently completed Olmesartan Reducing Incidence of Endstage Renal Disease in Diabetic Nephropathy Trial (ORIENT) and Olmesartan and Calcium Antagonists Randomized (OSCAR) studies will further define the role of ARBs in cardioprotection and renoprotection for high-risk patients.

Keywords: angiotensin receptor blockers, hypertension, outcomes, clinical trials

\section{Introduction}

Renin-angiotensin-aldosterone system (RAAS) blockade is a cornerstone of antihypertensive therapy in high-risk patients. ${ }^{1,2}$ Blockade of the RAAS reduces the risk of cardiovascular events and stroke in addition to providing renal protection. ${ }^{2,3}$ Angiotensin receptor blockers (ARBs) are among the agents recommended as first-line therapy for treatment of hypertension and reduction of cardiovascular risk. ${ }^{1,2}$

Prior to the ONgoing Telmisartan Alone and in combination with Ramipril Global Endpoint Trial (ONTARGET), the clinical relevance of the cardioprotective and renoprotective effects of ARBs, angiotensin-converting enzyme inhibitors (ACEIs), or their combination was largely unknown. ONTARGET, which evaluated 25,620 high-risk patients, provided a solid database to determine the benefits and risks of ARB and ACEI monotherapies as well as of combination therapy. ${ }^{4,5}$ This review article reassesses the role of $\mathrm{ARBs}$ in providing cardiovascular protection and the clinical implications of ONTARGET and similar trials.

\section{RAAS in pathogenesis of cardiovascular and renal disease}

The RAAS plays an important role in the pathophysiology of cardiovascular and renal disease. ${ }^{6,7}$ Its effects on the cardiovascular system include alterations in vascular 
reactivity, endothelial function, fibrosis, tissue remodeling, inflammation, and oxidative stress. ${ }^{6-8}$ In addition, renal disease (eg, microalbuminuria) is associated with endothelial dysfunction, accelerated atherosclerosis, and proteinuria, as well as with increased risk of cardiovascular morbidity, end-organ damage, and death. ${ }^{9-11}$

Apart from lowering blood pressure, blockade of the RAAS may provide independent effects on end-organ protection. ${ }^{12}$ Treatment with ACEIs and ARBs has demonstrated cardiorenal protective properties with favorable effects on blood pressure, renal hemodynamics, and proteinuria. ${ }^{13}$ For example, agents that block the RAAS pathway attenuate intraglomerular pressure and improve endothelial function in resistant arterioles. ${ }^{10}$ Due to the phenomenon of ACE escape and complementary mechanisms of action, dual blockade with an ACEI and an ARB has been hypothesized to provide greater RAAS inhibition and, in turn, greater cardiorenal benefits. $^{12,13}$

\section{Cardiovascular protection with ARBs}

The cardioprotective effects of ARBs were evident with the first ARB, losartan, as shown in a key pivotal trial, the Losartan Intervention For Endpoint Reduction (LIFE) study. ${ }^{14}$ The LIFE study was conducted to determine whether losartan was more effective than atenolol, a $\beta$-blocker, for the reduction of cardiovascular mortality and morbidity in hypertensive patients. This study was pivotal in establishing the role of angiotensin II blockade in cardiovascular protection. Losartan was better than atenolol in reducing the primary endpoint of cardiovascular death, stroke, or myocardial infarction by $14.6 \%$ $(P=0.009)$, and losartan was associated with a $25 \%$ lower incidence of new-onset diabetes, a significant cardiovascular risk factor. The cardiovascular protective effects were even more impressive in a subanalysis of 1195 diabetic patients enrolled in the LIFE study. ${ }^{15}$ This analysis established the efficacy of an ARB over that of a $\beta$-blocker in diabetic patients for cardiovascular protection, with a relative risk of 0.76 for the primary endpoint of cardiovascular death, stroke, or myocardial infarction, in favor of losartan $(P=0.031)$.

This cardiovascular protective effect was shown in even higher risk patients in the Morbidity and Mortality After Stroke, Eprosartan Compared With Nitrendipine for Secondary Prevention (MOSES) study. The MOSES study was the first to demonstrate superiority of an ARB over a calcium antagonist for secondary stroke prevention. ${ }^{16}$ The ARB, eprosartan, was shown to be superior to nitrendipine for the secondary prevention of morbidity and mortality after stroke in 1352 evaluable high-risk hypertensive patients with a history of a cerebral event within the previous 24 months, while having comparable reductions in blood pressure. The composite endpoint of mortality and all cardiovascular and cerebrovascular events, including recurrent events, was lower in patients treated with eprosartan versus nitrendipine (13.3 versus $16.7 / 100$ patient-years; $P=0.014$ ).

It has become clear in the last two decades that effective blockade of the RAAS confers important benefits in patients at high risk for cardiovascular disease. Earlier, ACE inhibitors were shown to confer target organ protection in patients with hypertension and diabetes; more recently, ARBs in large clinical trials have been shown to reduce the risk of cardiovascular, renal, and neurological complications.

ONTARGET resolved the question regarding the effectiveness of an ARB compared with an ACEI in high-risk patients with cardiovascular disease or diabetes mellitus without heart failure, ${ }^{17}$ although there continues to be confusion over the clinical implications of these findings. In ONTARGET, telmisartan was shown to be equivalent to ramipril in the incidence of primary outcome of death from cardiovascular causes, myocardial infarction, stroke, and hospitalization for heart failure (Figure 1). ${ }^{17}$ To underscore their similarities, the primary outcome occurred in $16.5 \%, 16.7 \%$, and $16.3 \%$ of patients receiving ramipril alone, telmisartan alone, or their combination, respectively. The secondary outcome in ONTARGET, death from cardiovascular causes, myocardial infarction, or stroke, occurred in $14.1 \%, 13.9 \%$, and $14.1 \%$ of patients receiving ramipril alone, telmisartan alone, or their combination, respectively. The relative risk ratio was 1.01 for the primary outcome and 0.99 for the secondary outcome with telmisartan versus ramipril, suggesting similarities in the degree of

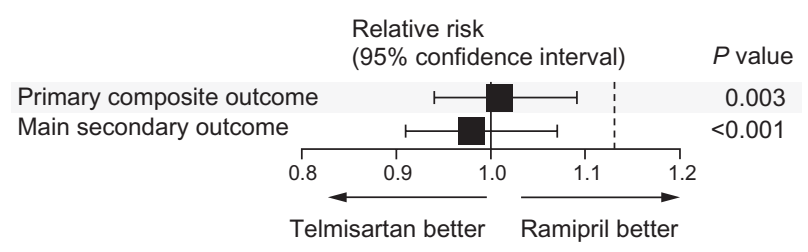

Figure I Comparison of telmisartan and ramipril for the relative risk of the primary and secondary outcomes of ONTARGET (ONgoing Telmisartan Alone and in combination with Ramipril Global Endpoint Trial). The primary composite outcome was death from cardiovascular causes, myocardial infarction, stroke, or hospitalization for heart failure. The main secondary outcome was death from cardiovascular causes, myocardial infarction, or stroke. The $P$ value is for the comparison with the noninferiority margins.

Copyright (C) 2008. Reproduced with permission from ONTARGET Investigators; Yusuf S, Teo KK, Pogue J, et al. Telmisartan, ramipril, or both in patients at high risk for vascular events. N Engl J Med. 2008;358: I547-1559. ${ }^{17}$ 
cardioprotection offered by these agents individually and in combination over ramipril alone. ${ }^{17}$

Furthermore, data from ONTARGET do not support the use of the combination of an ARB plus an ACEI. ${ }^{17}$ The relative risk ratios of combination therapy compared with ramipril were 1.00 for the primary outcome and 0.99 for the secondary outcome. Blood pressure reductions were greatest in the combination therapy group, with an average reduction at six weeks of 9.8/6.3 $\mathrm{mmHg}$ compared with $6.4 / 4.3 \mathrm{mmHg}$ for ramipril alone and 7.4/5.0 $\mathrm{mmHg}$ for telmisartan alone. However, no correlation of risk reduction was demonstrated in ONTARGET despite lower blood pressure in the combination group. Combination therapy was also associated with a higher incidence of adverse events.

Similar to ONTARGET, the Telmisartan Randomized AssessmeNt Study in aCE INtolerant subjects with cardiovascular Disease (TRANSCEND) trial was conducted with a population of patients intolerant to ACEIs with established coronary artery, peripheral vascular, or cerebrovascular disease, or diabetes with end-organ damage. ${ }^{18}$ TRANSCEND showed a statistically significant benefit of telmisartan versus placebo for the secondary endpoint of cardiovascular death, myocardial infarction, and stroke (hazard ratio [HR] 0.87 ; $P=0.048) .{ }^{18}$ Compared with placebo, telmisartan was also associated with fewer cases of left ventricular hypertrophy and cardiovascular-related hospitalizations.

Another recently completed study, the Olmesartan and Calcium Antagonists Randomized (OSCAR) study, compared olmesartan (an ARB) alone with the combination of olmesartan and a calcium channel blocker in elderly high-risk Japanese patients with hypertension. ${ }^{19}$ Primary endpoints include the composite of fatal and nonfatal cardiovascular events, and death from any cause. Data from this study will provide further insight into the role of ARBs and combination therapy.

It remains to be shown whether there are some patient subgroups, such as those with diabetes and proteinuria, who may benefit from dual inhibition of RAAS. For now, in uncomplicated hypertension, dual blockade of RAAS is not indicated.

\section{Long-term ARB tolerability and renoprotection}

Early clinical trials suggested the efficacy of ARBs in the prevention of progression of nephropathy in diabetic patients. For example, the Irbesartan in Patients with Type 2 Diabetes and Microalbuminuria (IRMA) study demonstrated that at an optimal dose of $300 \mathrm{mg}$, irbesartan reduced the rate of progression to clinical albuminuria (HR $0.30 ; P<0.001)$ and restored normoalbuminuria in a significant proportion of 590 patients with hypertension and type 2 diabetes. ${ }^{20}$ Similarly, the Irbesartan Diabetic Nephropathy Trial (IDNT) was conducted to determine whether an ARB or a calcium channel antagonist would provide protection against advancing nephropathy in 1715 hypertensive patients with type 2 diabetes beyond that of hypertension control. ${ }^{21}$ Compared with placebo and amlodipine, irbesartan had a $20 \%(P=0.02)$ and $23 \%(P=0.006)$ lower risk of a doubling of the baseline serum creatinine concentration, development of end-stage renal disease, or death from any cause.

ONTARGET showed a better tolerability profile of telmisartan versus ramipril, with fewer cases of angioedema (relative risk $[\mathrm{RR}] 0.4 ; P<0.01$ ) and cough (RR 0.26; $P<0.001)$ but a higher rate of hypotensive symptoms (RR 1.54; $P<0.001) .{ }^{17}$ These data are consistent with those from previous studies, including a systematic review of 61 clinical trials. ${ }^{22}$ A metaanalysis of randomized, controlled trials revealed cough rates of $9.9 \%$ in ACEI-treated patients compared with $3.2 \%$ in ARB-treated patients. ${ }^{22}$ Lower rates were observed in cohort studies, but the cough rate was consistently lower in ARBtreated patients. ${ }^{22}$ In three studies reporting angioedema, all reported cases occurred in patients receiving an ACEI. ${ }^{22}$

Analysis of renal outcomes in ONTARGET showed equivalence between telmisartan and ramipril for effects on kidney function, including the primary renal outcomes of dialysis, doubling of serum creatinine, and death (Figure 2 ). ${ }^{23}$ ONTARGET demonstrated that combination treatment with telmisartan and ramipril reduced albuminuria compared with ramipril (HR $0.88 ; P=0.003$ ) but was associated with increased risk of renal impairment and acute dialysis in highrisk patients with diabetes and vascular diseases. ${ }^{23}$ Compared with ramipril alone, the combination of telmisartan and ramipril resulted in a higher incidence of renal dysfunction, doubling of serum creatinine, need for dialysis, and incidence of hyperkalemia. ${ }^{17,23}$

Similarly, during the TRANSCEND study, treatment with telmisartan was well tolerated. ${ }^{18}$ Fewer patients discontinued treatment with telmisartan than with placebo, despite more patients experiencing mild hypotensive symptoms. ${ }^{18} \mathrm{~A}$ recent analysis of renal outcomes for this study demonstrated that telmisartan was similar to placebo in renal outcomes, including the composite of doubling of serum creatinine and dialysis, and did not offer any significant renoprotective benefits. ${ }^{24}$ Telmisartan was shown to increase the incidence of the composite outcome in patients with no 


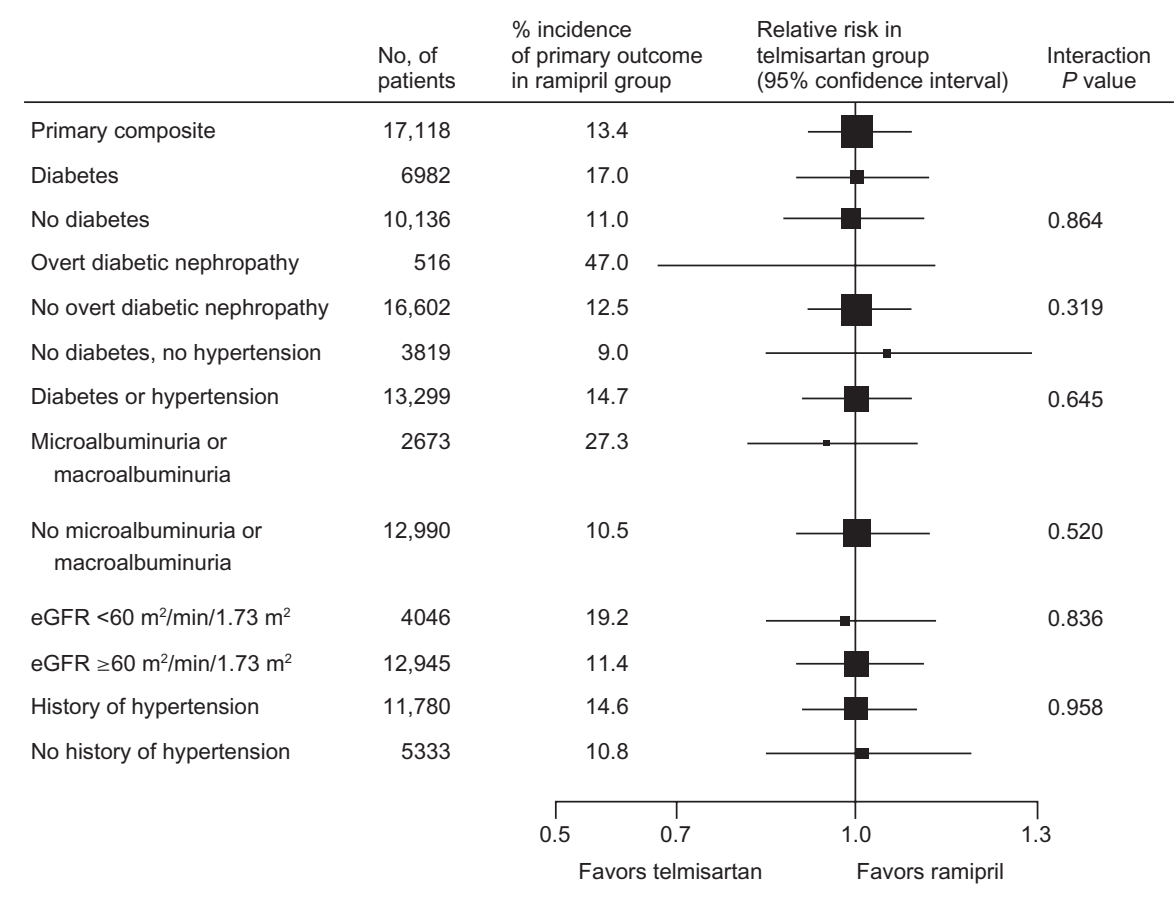

Figure 2 Comparison of telmisartan and ramipril for the relative risk of the primary renal outcomes of dialysis, doubling of serum creatinine, and death in subgroups of ONTARGET (ONgoing Telmisartan Alone and in combination with Ramipril Global Endpoint Trial) patients.

Reprinted from The Lancet, with permission from Elsevier. Mann JF, Schmieder RE, McQueen MJ, et al; TRANSCEND (Telmisartan Randomised Assessment Study in ACE Intolerant Subjects with Cardiovascular Disease) Investigators. Renal outcomes with telmisartan, ramipril, or both, in people at high vascular risk (the ONTARGET study): A multicentre, randomised, double-blind, controlled trial. Lancet. 2008;372:547-553. ${ }^{23}$

Abbreviation: eGFR, estimated glomerular filtration rate.

microalbuminuria or an estimated glomerular filtration rate (eGFR) of $\geq 60 \mathrm{~mL} / \mathrm{min} / 1.73 \mathrm{~m}^{2}$. However, this outcome was reduced in patients receiving telmisartan who had microalbuminuria or an eGFR $<60 \mathrm{~mL} / \mathrm{min} / 1.73 \mathrm{~m}^{2} .^{24}$

The recently completed Olmesartan Reducing Incidence of Endstage Renal Disease in Diabetic Nephropathy Trial (ORIENT), a randomized, double-blind, placebo-controlled Phase III study, examined the effects of olmesartan on the rate of progression of diabetic nephropathy. Participants included patients in Japan and Hong Kong, aged 30 to 70 years, with type 2 diabetes mellitus. ${ }^{25,26}$ In this study, 577 patients were randomized to receive olmesartan $10-40 \mathrm{mg}$ or placebo. The primary efficacy measure was a composite of the time to first occurrence of doubling of serum creatinine, end-stage renal disease, or death. Secondary efficacy endpoints included a composite of the time to first occurrence of a cerebrovascular/ cardiovascular event, change in proteinuria, and change in renal function calculated by the slope of the reciprocal of serum creatinine against time. Patients receiving an ACEI prior to enrollment were allowed to continue taking the same dose of the ACEI, with $72 \%$ of enrolled patients receiving combination therapy with an ACEI and olmesartan, and a median follow-up of three years. ${ }^{25}$ Results of ORIENT are not yet published.

\section{Conclusion}

Data from ONTARGET and TRANSCEND establish that telmisartan is as effective as ramipril. A growing body of evidence demonstrating the effectiveness and long-term cardiorenal protective properties of ARBs indicate that ARBs may be used successfully and with potentially greater clinical benefit compared with ACEIs. Additionally, the evidence suggests that each ARB may have unique properties that go beyond the established "class effect". However, further investigation is needed to clarify these findings. ONTARGET data demonstrate that combination therapy with an ARB and an ACEI does not provide an additive benefit and, therefore, should not be used routinely in clinical practice, particularly in high-risk and elderly patients.

\section{Disclosure}

This work was supported by Boehringer Ingelheim Pharmaceuticals. Writing and editorial assistance was also funded by Boehringer Ingelheim Pharmaceuticals. The author is on the speakers' bureaus of Cogenix, Primed, Medcom, and AHM, which facilitate educational programs for various pharmaceutical companies, but received no compensation related to the development of the manuscript. 


\section{References}

1. Rosendorff C, Black HR, Cannon CP, et al; American Heart Association Council for High Blood Pressure Research; American Heart Association Council on Clinical Cardiology; American Heart Association Council on Epidemiology and Prevention. Treatment of hypertension in the prevention and management of ischemic heart disease: A scientific statement from the American Heart Association Council for High Blood Pressure Research and the Councils on Clinical Cardiology and Epidemiology and Prevention. Circulation. 2007;115:2761-2788.

2. Chobanian AV, Bakris GL, Black HR, et al; National Heart, Lung, and Blood Institute Joint National Committee on Prevention, Detection, Evaluation, and Treatment of High Blood Pressure; National High Blood Pressure Education Program Coordinating Committee. The Seventh Report of the Joint National Committee on Prevention, Detection, Evaluation, and Treatment of High Blood Pressure: The JNC 7 report. JAMA. 2003;289:2560-2572.

3. Lip GY, Beevers DG. More evidence on blocking the renin-angiotensinaldosterone system in cardiovascular disease and the long-term treatment of hypertension: Data from recent clinical trials (CHARM, EUROPA, ValHEFT, HOPE-TOO and SYST-EUR2). J Hum Hypertens. 2003; 17:747-750.

4. Teo K, Yusuf S, Sleight P, et al; ONTARGET/TRANSCEND Investigators. Rationale, design, and baseline characteristics of 2 large, simple, randomized trials evaluating telmisartan, ramipril, and their combination in high-risk patients: the Ongoing Telmisartan Alone and in Combination with Ramipril Global Endpoint Trial/Telmisartan Randomized Assessment Study in ACE Intolerant Subjects with Cardiovascular Disease (ONTARGET/TRANSCEND) trials. Am Heart J. 2004;148:52-61.

5. Sleight P. The ONTARGET/TRANSCEND Trial Programme: Baseline data. Acta Diabetol. 2005;42 Suppl 1:S50-S56.

6. Paul M, Poyan Mehr A, Kreutz R. Physiology of local renin-angiotensin systems. Physiol Rev. 2006;86:747-803.

7. Atlas SA. The renin-angiotensin aldosterone system: Pathophysiological role and pharmacologic inhibition. JManag Care Pharm. 2007;13 Suppl B: 9-20.

8. Min LJ, Mogi M, Iwanami J, et al. Cross-talk between aldosterone and angiotensin II in vascular smooth muscle cell senescence. Cardiovasc Res. 2007;76:506-516.

9. Olsen MH, Wachtell K, Hermann KL, et al. Left ventricular hypertrophy is associated with reduced vasodilatory capacity in the brachial artery in patients with longstanding hypertension. A LIFE substudy. Blood Press. 2002;11:285-292.

10. Montalescot G, Collet JP. Preserving cardiac function in the hypertensive patient: Why renal parameters hold the key. Eur Heart J. 2005;26:2616-2622.

11. Gerstein HC, Mann JF, Yi Q, et al. Albuminuria and risk of cardiovascular events, death, and heart failure in diabetic and nondiabetic individuals. JAMA. 2001;286:421-426.

12. Weir MR. Effects of renin-angiotensin system inhibition on end-organ protection: Can we do better? Clin Ther. 2007;29:1803-1824.

13. Parving $\mathrm{HH}$, Andersen S, Jacobsen $\mathrm{P}$, et al. Angiotensin receptor blockers in diabetic nephropathy: Renal and cardiovascular end points. Semin Nephrol. 2004;24:147-157.

14. Dahlof B, Devereux RB, Kjeldsen SE, et al. Cardiovascular morbidity and mortality in the Losartan Intervention For Endpoint reduction in hypertension study (LIFE): A randomised trial against atenolol. Lancet. 2002;359:995-1003.

Vascular Health and Risk Management

\section{Publish your work in this journal}

Vascular Health and Risk Management is an international, peerreviewed journal of therapeutics and risk management, focusing on concise rapid reporting of clinical studies on the processes involved in the maintenance of vascular health; the monitoring, prevention and treatment of vascular disease and its sequelae; and the involvement of
15. Lindholm LH, Ibsen H, Dahlof B, et al. Cardiovascular morbidity and mortality in patients with diabetes in the Losartan Intervention For Endpoint reduction in hypertension study (LIFE): A randomised trial against atenolol. Lancet. 2002;359:1004-1010.

16. Schrader J, Luders S, Kulschewski A, et al. Morbidity and mortality after stroke, eprosartan compared with nitredipine for secondary prevention: Principal results of a prospective randomized controlled study (MOSES). Stroke. 2005;36:1218-1224.

17. ONTARGET Investigators; Yusuf S, Teo KK, Pogue J, et al. Telmisartan, ramipril, or both in patients at high risk for vascular events. N Engl J Med. 2008;358:1547-1559.

18. Telmisartan Randomised AssessmeNt Study in ACE iNtolerant subjects with cardiovascular Disease (TRANSCEND) Investigators; Yusuf S, Teo K, Anderson C, et al. Effects of the angiotensin-receptor blocker telmisartan on cardiovascular events in high-risk patients intolerant to angiotensin-converting enzyme inhibitors: A randomised controlled trial. Lancet. 2008;372:1174-1183.

19. Ogawa H, Kim-Mitsuyama S, Jinnouchi T, et al. Rationale, design and patient baseline characteristics of OlmeSartan and calcium antagonists randomized (OSCAR) study: A study comparing the incidence of cardiovascular events between high-dose angiotensin II receptor blocker (ARB) monotherapy and combination therapy of ARB with calcium channel blocker in Japanese elderly high-risk hypertensive patients (ClinicalTrials. gov no. NCT00134160). Hypertens Res. 2009;32: 575-580.

20. Parving H-H, Lehnert H, Brochner-Mortensen J, et al. The effect of irbesartan on the development of diabetic nephropathy in patients with type 2 diabetes. N Engl J Med. 2001;345:870-878.

21. Lewis E, Hunsicker LG, Clarke WR, et al. Renoprotective effect of the angiotensin-receptor antagonist irbesartan in patients with nephropathy due to type 2 diabetes. $N$ Engl J Med. 2001;345:851-860.

22. Matchar DB, McCrory DC, Orlando LA, et al. Systematic review: comparative effectiveness of angiotensin-converting enzyme inhibitors and angiotensin II receptor blockers for treating essential hypertension. Ann Intern Med. 2008;148:16-29.

23. Mann JF, Schmieder RE, McQueen MJ, et al; TRANSCEND (Telmisartan Randomised Assessment Study in ACE Intolerant Subjects with Cardiovascular Disease) Investigators. Renal outcomes with telmisartan, ramipril, or both, in people at high vascular risk (the ONTARGET study): A multicentre, randomised, double-blind, controlled trial. Lancet. 2008;372:547-553.

24. Mann JF, Schmieder RE, Dyal L, et al; TRANSCEND (Telmisartan Randomised Assessment Study in ACE Intolerant Subjects with Cardiovascular Disease) Investigators. TRANSCEND Investigators. Effect of telmisartan on renal outcomes: A randomized trial. Ann Intern Med. 2009;151:1-10.

25. Imai E, Chan J, Ito S, Haneda M, Mankino H. Effects of olmesartan on renal and cardiovascular outcomes in type 2 diabetic patients with overt nephropathy [Abstr SA764]. Presented at World Congress of Nephrology, May 23, 2009, Milan, Italy.

26. ORIENT: Olmesartan reducing incidence of end stage renal disease in diabetic nephropathy trial. Available at: www.clinicaltrials.gov/ ct $2 /$ show $/$ NCT00141453? term $=$ NCT00141453\&rank $=$. Accessed December 9, 2009.

metabolic disorders, particularly diabetes. This journal is indexed on PubMed Central and MedLine. The manuscript management system is completely online and includes a very quick and fair peer-review system, which is all easy to use. Visit http://www.dovepress.com/ testimonials.php to read real quotes from published authors. 